\title{
Parametric and Non Parametric Time-Frequency Analysis of Biomedical Signals
}

\author{
S. Elouaham, R. Latif, A. Dliou \\ ESSI, National School of applied \\ Sciences, Ibn Zohr University \\ Agadir, Morocco
}

\author{
M. LAABOUBI \\ High School of technology \\ Ibn Zohr University \\ Guelmim, Morocco
}

\author{
F. M. R. Maoulainie \\ Team of Child, Health and \\ Development, CHU, Faculty of \\ Medicine, Cadi Ayyad University, \\ Marrakech, Morocco
}

\begin{abstract}
Due to non-stationary multicomponent nature of the electrocardiogram (ECG) signal, its analysis by the monodimensional techniques, temporal and frequenctial, can be very difficult. The use of the time-frequency techniques can be inevitable to achieve to a correct diagnosis. Between the different existing parametric and non-parametric time-frequency techniques, the Periodgram, Capon, Choi-Williams and Smoothed Pseudo Wigner-Ville were chosen to deal with analysis of this biomedical signal. In a first time, a comparison between these time-frequency techniques was made by analyzing modulated signal to make in evidence the technique that gives a good resolution and low level of cross-terms. In a second time, the Periodogram which presents a powerful technique was applied to a normal and abnormal ECG signal. The results show the effectiveness of this time-frequency in analyzing this type of biology signal.
\end{abstract}

Keywords- ECG; Time-frequency; Periodogram; Capon; Choiwilliams.

\section{INTRODUCTION}

The electrocardiogram (ECG) is one of the major physiological signals generated from heart's rhythmic polarization and depolarization. The $\mathrm{P}$ and $\mathrm{T}$ waves and QRS complex are the principles components of this biological signal. The $\mathrm{P}$ wave presents the arterial activation, the QRS complex reflects the ventricular depolarization and $\mathrm{T}$ wave refers to the ventricular repolarization $[1,2]$. These different ECG components have specific shapes in the time and frequency domains, which allow the specialists to detect possible existing anomalies only by analyzing ECG signal in these two domains. The electrocardiograms signals are generally non-stationary. The information giving by using the Fourier transform does not provide any indication about the frequency notion over time. Otherwise, the temporal domain presents the weakness that the frequency component which presents the pertinent information is not visualized. To exceed the one-dimensional limitations, the use of the time-frequency techniques is inevitable; these techniques provide an accurate description of the non-stationary behavior of the ECG signals, as a conjoint representation, in time and frequency. The timefrequency techniques used are able to present the evolution of ECG signal power as a function of both time and frequency. In this study, the two major categories of the time-frequency techniques are utilized, the parametric and the non-parametric ones. The chosen time-frequency techniques are selected for their interesting proprieties [1-15]. The used time-frequency techniques are: Periodogram, Capon, Choi-williams and Smoothed pseudo Wigner-Ville. A comparison based on the variance and the resolution factors of some modulated signals are proposed to select the most suitable technique, which will be more adequate for analyzing the ECG signal. In this paper, the work was based for analyzing a normal and an abnormal ECG with some types of arrhythmia pathology. Cardiac arrhythmias are divided into two groups, the first one is life threatening and requires immediate treatment with an automated external defibrillator (AED) or an implantable cardioverter defibrillator (ICD) $[16,17]$. The second one is not deadly, but requires treatment sustainability. The abnormal ECG signals used in this work are obtained from patients with cu ventricular tachyarrhythmia and malignant ventricular arrhythmia.

The Periodogram performance surpass others techniques in order to reducing the cross terms and increasing the resolution. The cross-terms present the principle problem of the timefrequency which can obscure some important information in the time-frequency plan.

The article is organized as the follows, firstly we will present, in the section II, the different techniques used in this work. Secondly, in the section III, we will detail the biomedical signals used in this study. Thirdly, the section IV and V will show the main obtained results. Finally, we will conclude with a conclusion in the section VI.

\section{TIME-FREQUENCY TECHNIQUES}

\section{A. Parametric techniques}

The parametric time-frequency techniques used in this work are the Capon, the Periodogram.

\section{Capon distribution}

The estimator of minimum variance called Capon estimator (CA) does not impose a model on the signal. At each frequency $\mathrm{f}$, this method seeks a matched filter whose response is 1 for the frequency $f$ and 0 everywhere else [3-7].

$$
\begin{array}{r}
C A(n, f)=a(n, f)^{H} R_{x} a(n, f) \\
=\frac{1}{Z_{f}^{H} \cdot R_{x}[n]^{-1} \cdot Z_{f}}
\end{array}
$$


Where:

- $C A(n, f)$ is the output power of the filter Capon, excited by the discrete signal $\mathrm{x}(\mathrm{n})$ sampled at the period te,

- $a(n, f)=\left(a_{\mathrm{O}}, \ldots, a_{p}\right)$ is the impulse response of the filter at frequency $\mathrm{n}$,

- $R_{x}[n]=E\left\{x[n] x^{T}[n]\right\}$ is the autocorrelation matrix of crossed $\mathrm{x}(\mathrm{n})$ of dimension $(p+1) *(p+1)$,

- $x[n]=(x(n-p), \ldots, x(n))$ is the signal at time $\mathrm{n}$,

- $z_{f}^{H}=\left(1, e^{2 i \pi f t_{e}}, \ldots, e^{2 i \pi f t e} p\right)$ is the steering vector,

- $(p+1)$ is the number of filter coefficient and the exponent $\mathrm{H}$ for conjugate transpose and the superscript $\mathrm{T}$ for transpose.

\section{Periodogram technique}

The Periodogram (PE) is the derivate of the Capon (CA) technique. The spectral estimator of this method is defined by the following equation [3-7]:

$$
P E(n, f)=Z_{f}^{H} \cdot R_{x} \cdot Z_{f} /\left((\mathrm{p}+1)^{2}\right)
$$

The two previous techniques defined by the equations 1 and 2 can be applied sliding windows. There is no theoretical criterion for choosing the filter order and duration of the window [4]. The parametric techniques depend on the signal so that the frequency response has a different shape and then different properties according to the signal characteristics. The choice of the window is more crucial to the time-frequency resolution. CA and PE estimator usually has a better frequency resolution. Both techniques are well suited to signals containing some strong spectral components such as ECG biomedical signals.

\section{B. Non parametric techniques}

1. Smoothed Pseudo Wigner-Ville

The majority of the non parametric time-frequency representations are represented by the cohen class [7-15]. This class includes inter alias the Wigner-Ville distributions. Among the large number of existing time-frequency representations some authors have proposed using the Wigner-Ville $[9,10]$. To avoid the covering of frequential components in the timefrequency representation, in this study we use in the place of the real signal $x(t)$ the analytical signal $x_{a}(t)$ defined by the expression:

$$
x_{a}(t)=x(t)+i H\{x(t)\}
$$

Where $\mathrm{i}^{2}=-1$ and $x(t)$ is the signal with real values and $H\{x(t)\}$ it's Hilbert transform.

The distribution of Wigner-Ville associated to a signal $x(t)$ , of finished energy, is the function $W_{x}(t, f)$ depending of the temporal (t) and frequential (f) parameters. This distribution is given by the following expression:

$$
W_{x}(t, f)=\int_{-\infty}^{+\infty} x\left(t+\frac{\tau}{2}\right) \cdot x^{*}\left(t-\frac{\tau}{2}\right) e^{-2 i \pi f \tau} d \tau
$$

Where $x^{*}$ indicates the complex conjugate of ${ }_{x}(t)$.

The transform called the Smoothed Pseudo Wigner-Ville (SPWV) is implanted in this work to attenuate the interference terms presented between the inner components figured in Wigner-Ville representation which decrease the visibility of the time-frequency image. The SPWV use two smoothing windows $\mathrm{h}(\mathrm{t})$ and $\mathrm{g}(\mathrm{t})$. These smoothing windows are introduced into the Wigner-Ville distribution definition in order to allow a separate control of interference either in time $(\mathrm{g})$ or in frequency (h). The expression of this representation is defined by [12]

$$
\begin{aligned}
\operatorname{SPWV} V_{x}(t, f) & =\int_{-\infty}^{+\infty} h\left|\left(\frac{\tau}{2}\right)^{2}\right| \int_{-\infty}^{+\infty} g(t-u) x\left(u+\frac{\tau}{2}\right) \\
. & *\left(u-\frac{\tau}{2}\right) e^{-2 i \pi f \tau} d u d \tau
\end{aligned}
$$

Where $\mathrm{h}(\mathrm{t})$ is a smoothing frequential window and $\mathrm{g}(\mathrm{t})$ is a smoothing temporal window.

\section{Choi-Williams technique}

The Choi-Williams distribution was developed as a method of finding the Wigner-Ville Distribution with the minimum amount of cross-term interference. The Choi-Williams distribution is a shift-invariant transform. Essentially, the distribution is a smoothed version of the Wigner-Ville distribution through a kernel function defined by [15-17]:

$$
f(\varepsilon, \tau)=\exp \left[-\frac{(\pi \varepsilon \tau)^{2}}{2 \sigma^{2}}\right]
$$

The Choi-Williams distribution is then defined as:

$$
C W_{x}(t, f)=\sqrt{\frac{2}{\pi}} \int_{-\infty}^{+\infty} \frac{\sigma}{|\tau|} \exp ^{-2 \sigma^{2}(s-t)^{2} / \tau^{2}} A_{x} \exp ^{-j 2 \pi f \tau} d s d \tau
$$

Where

$$
A_{x}=x\left(s+\frac{\tau}{2}\right) x^{*}\left(s-\frac{\tau}{2}\right)
$$

The smoothing of the distribution is controlled by the constant $\sigma$. As $\sigma \rightarrow \infty$, the Choi-Williams (CW) distribution will simplify converges to the Wigner-Ville distribution, as the kernel goes to 1 . The cross-terms are reduced with smaller values of $\sigma$. The Choi-Williams distribution is a bilinear timefrequency representation [17].

\section{BIOMEDICAL SIGNALS}

\section{A. Normal ECG signal}

The normal signal ECG is characterized with $\mathrm{P}$ wave, Twave, and complex the QRS. The figure 1 shows an example of a normal ECG signal. 


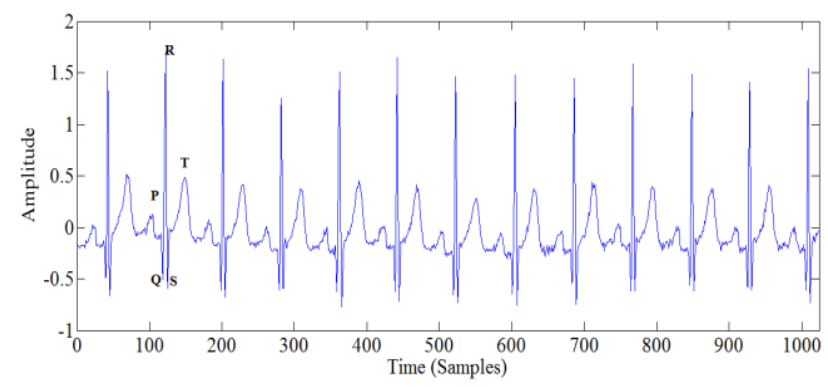

Figure.1. Normal ECG signal

\section{B. Abnormal signal}

The abnormal signals used in this work are cu ventricular tachyarrhythmia, and malignant ventricular arrhythmia. The figure 2 represents misalignments of the third QRS complex of cu ventricular tachyarrhythmia.

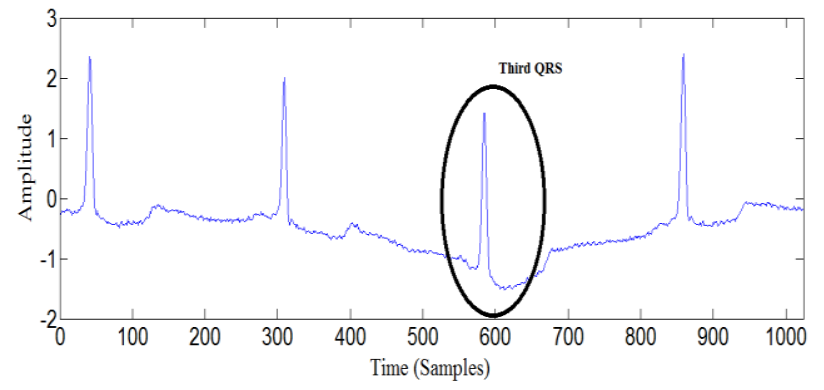

Figure. 2. Abnormal ECG signal with cu ventricular tachyarrhythmia

And the last anomaly is malignant ventricular arrhythmia presented in figure 3. The T-wave signal has disappeared and the QRS complexes are wide.

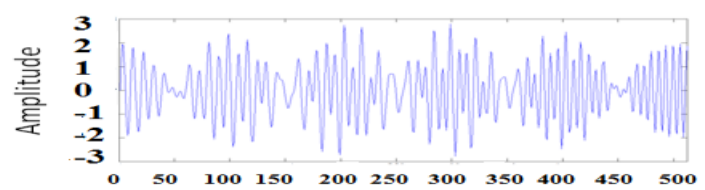

a
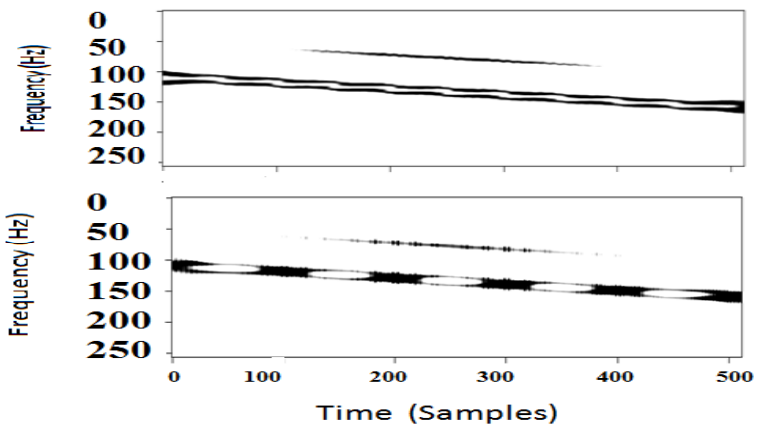

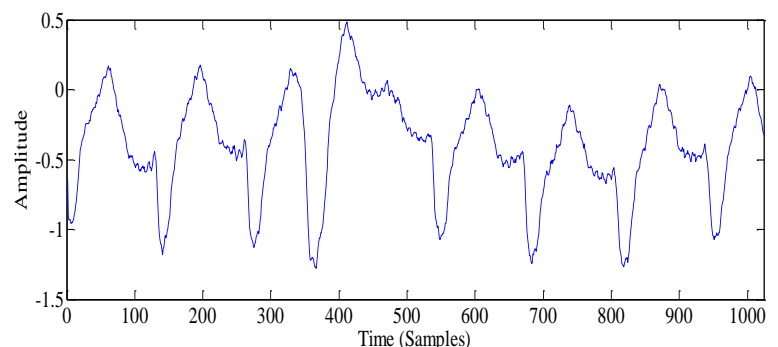

Figure. 3. Abnormal ECG signal of malignant ventricular

The ECG signals were provided by MIT-BIH database [18].

\section{PERFORMANCE OF TIME-FrEQUENCY TECHNIQUES}

\section{A. Modulated signals}

The time-frequency techniques are applied in this section to the signal given by the following equation,

$$
\begin{aligned}
\mathrm{x}(\mathrm{t})= & \sin \left(2 \pi\left(f_{1}+50 t\right) \mathrm{t}\right)+\sin \left(2 \pi\left(f_{2}+50 t\right) \mathrm{t}\right)+ \\
& \sin (2 \pi \mathrm{t}) \sin \left(2 \pi\left(f_{3}+50 t\right) \mathrm{t}\right)
\end{aligned}
$$

Where

$$
f_{1}=50, f_{2}=100, f_{3}=110 ;
$$

The signal used is composed of three components; two components of high frequency and the third component is of low frequency .The goal of this application is the choice of appropriate method for specific application
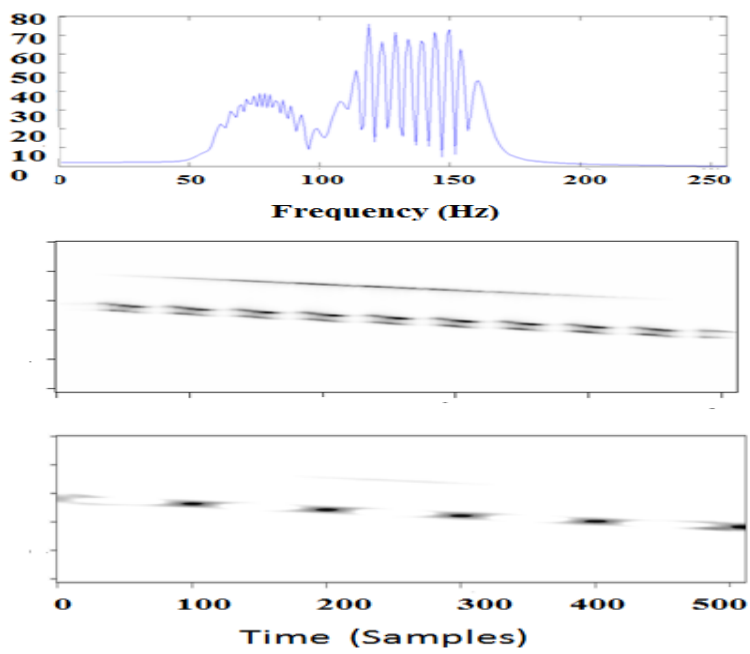

Figure. 4. Modulated signal (a), Fourier spectrum (b), PE (c), CA (d), CW (e), SPWV (f) Resultants

The figure $4 \mathrm{a}$ presents the modulated signal used for the comparison between the time-frequency techniques. The Fourier transform spectrum presented in figure $4 \mathrm{~b}$ shows the evolution of frequency content from 50 to 165 , and cannot give any information about number of the components frequency and their change over time. The figure $4 \mathrm{c}$ presented by periodogram gives a good localization of the two high frequency components than the others techniques. This technique can separate clearly the frequency components. The non parametric time-frequency images, figures $4 \mathrm{e}$ and $4 \mathrm{f}$, show the presence of high level of cross-terms and low degree of resolution which make the identification of the two frequency components more delicate than the periodogram technique. 


\section{B. Variance}

The time-frequency techniques used in this study are applied to a monocomponent signal to find the most performance technique.

The monocomponent signal used is given by the following equation:

$$
x(t)=a e^{j \phi(t)}
$$

The instantaneous frequency (IF) is given by the following equation:

$$
f=\frac{1}{2 \pi} d \phi / d t=f_{0}+\beta t
$$

Where $\mathrm{a}=1, f_{\mathrm{o}}=0.05 f_{s}, \beta=0.4 \mathrm{f}_{s}, \phi(t)$ is the analytic signal phase and $f_{\mathrm{s}}=1 / \mathrm{T}$ is the sampling frequency.

The bias (B) and the variance (VAR) of the estimate present the most important factors that decide the quality of estimation. These two notions can be defined by the following expressions:

$$
\begin{aligned}
B\left(\begin{array}{c}
\Lambda \\
f_{i}(t)
\end{array}\right) & =\varepsilon\left[\begin{array}{c}
\Lambda \\
\Delta f_{i}(t)
\end{array}\right] \\
\operatorname{VAR}\left(\begin{array}{c}
\Lambda \\
f_{i}(t)
\end{array}\right) & =\varepsilon\left[\begin{array}{c}
\Lambda \\
\left(\Delta f_{i}(t)\right)
\end{array}\right]^{2}
\end{aligned}
$$

With:

$$
\Delta \stackrel{\Lambda}{f_{i}(t)}=f_{i}(t)-\stackrel{\Lambda}{f_{i}}(t)
$$

Where $f_{i}(t)$ and $\hat{f}_{i}(t)$ are the instantaneous frequency and instantaneous frequency estimate respectively. The signal length used in the time-frequency techniques is $\mathrm{N}=256$ samples and the total signal duration is $1 \mathrm{~s}$. The sampling frequency was $f_{\mathrm{s}}=2 \mathrm{NHz}$. Using different Signal-to-Noise Ratio (SNR), gaussian white noise samples are added to the signal. The figure 5 shows the performance of the PE, CA and SPWV, CW time-frequency techniques applied to a linear FM signal with 256 points.

The table 1 gives the variance values of the parametric (PE, $\mathrm{CA}$ ) and non-parametric (SPWV, CW) techniques. According to the results of this table, the Periodogram time-frequency technique has a minimal variance for all SNR's. The low minimum variance can indicate the performance of the timefrequency techniques. The PE technique surpasses the other time-frequency techniques in robustness where it gives the minimum variance at low SNR.

\section{RESUlTS AND DISCUSSION}

The figures 6 to 8 present the time-frequency images of the normal and abnormal ECG signals (figure 1 to 3 ). This time- frequency images are obtained by using the calculation of the equation 2 and 10 of the Periodogram and the Choi-Williams techniques respectively. The figures 6a (2D) and 6a' (3D) show the Periodogram images of the normal ECG signal (figure 1). By the same way, the figures $6 \mathrm{~b}(2 \mathrm{D})$ and 6b' (3D) expose the Choi-willams images of the same signal.

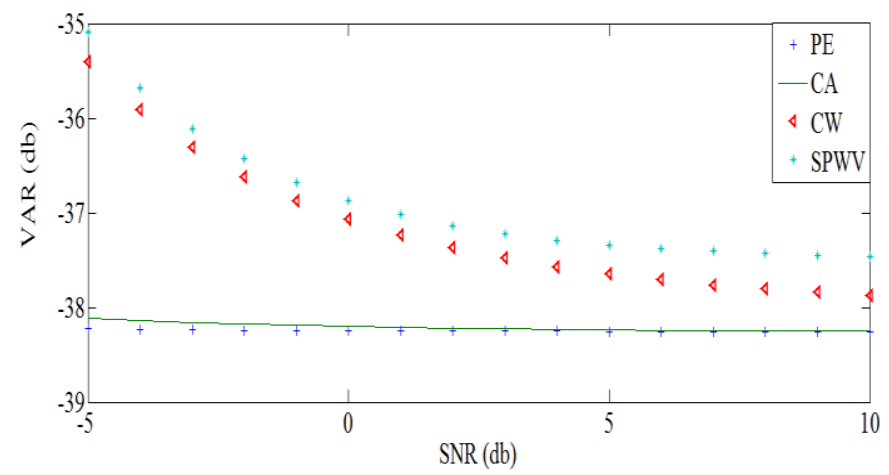

Figure.5. Performance of PE, CA, CW and SPWV in IF estimation of a linear FM signal with length $\mathrm{N}=256$ samples.

TABLE: VARIANCE OF PARAMETRIC AND NON PARAMETRIC TECHNIQUES

\begin{tabular}{|c|c|c|c|l|}
\hline SNR(db) & PE & CA & CW & SPWV \\
\hline-5 & & & & \\
\hline-4 & -38.2252 & -38.1106 & -35.4000 & -35.0960 \\
-3 & -38.2370 & -38.1379 & -35.9079 & -35.6796 \\
-2 & -38.2411 & -38.1598 & -36.3039 & -36.1092 \\
-1 & -38.2444 & -38.1776 & -36.6174 & -36.4273 \\
0 & -38.2471 & -38.2038 & -36.8683 & -36.6749 \\
1 & -38.2491 & -38.2136 & -37.2340 & -36.8680 \\
2 & -38.2507 & -38.2216 & -37.3674 & -37.0191 \\
3 & -38.2520 & -38.2282 & -37.4772 & -37.2246 \\
4 & -38.2530 & -38.2336 & -37.5682 & -37.2911 \\
5 & -38.2538 & -38.2381 & -37.6440 & -37.3417 \\
6 & -38.2544 & -38.2419 & -37.7074 & -37.3795 \\
7 & -38.2548 & -38.2451 & -37.7606 & -37.4081 \\
8 & -38.2552 & -38.2477 & -37.8052 & -37.4300 \\
9 & -38.2555 & -38.2500 & -37.8429 & -37.4473 \\
10 & -38.2556 & -38.2519 & -37.8749 & -37.4615 \\
\hline
\end{tabular}

The Periodogram time-frequency images (figure 6a et 6a') give a good location of the QRS complexes and the T waves. By the comparison of the periodogram results with those obtained by Choi-williams (Fig. 6 b and $6 b^{\prime}$ ), we note that the periodogram technique gives the best results. The figure 7 presents the time-frequency images of the abnormal ECG signal obtained from a patient with $\mathrm{cu}$ ventricular tachyarrhythmia (figure 2). The Periodogram time-frequency image (fig. 7a) gives us a good localization of QRS complexes with the transient's appearance in the third QRS complex. The visualization of these transients in the time-frequency image in the figure 7a' indicates the ability of detecting abnormalities by the Periodogram technique. The Periodogram technique can track the change in the frequency components of each QRS complex. The figures $7 \mathrm{~b}$ and $7 \mathrm{~b}$ ' represent the Choi-williams time-frequency images. These images give no information about the transients in the third QRS complex which present the existing abnormality. These obtained results expose the robustness of the Periodogram technique for identifying the pathology than the Choi-williams method. 

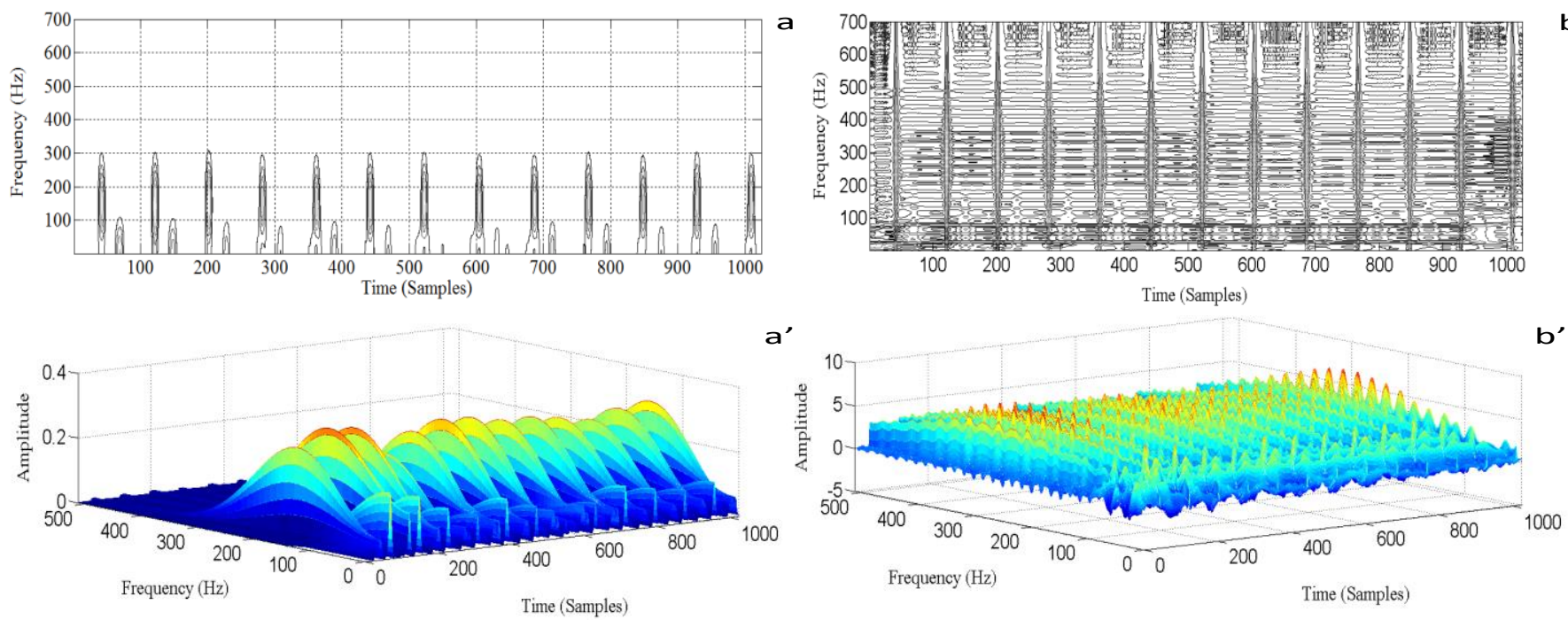

Figure.6. Periodogram (a and a') and Choi-williams (b and b') time-frequency images of a normal ECG signal.
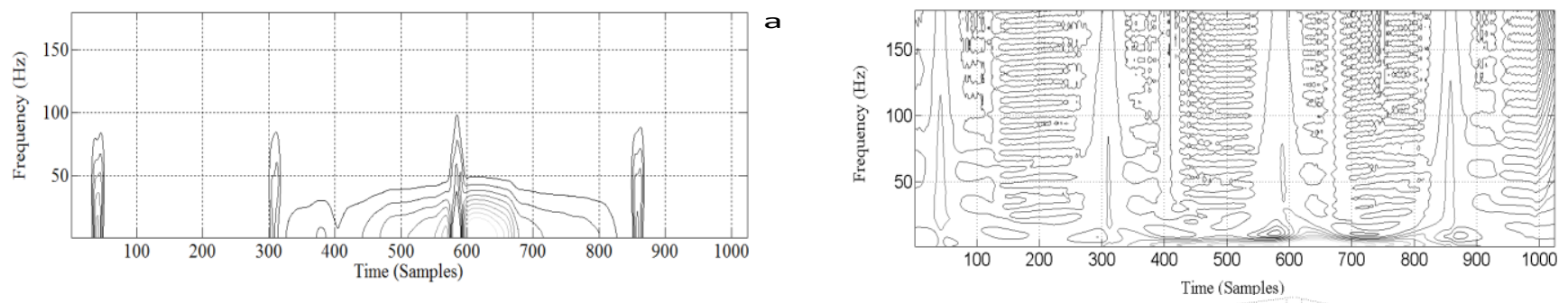

b
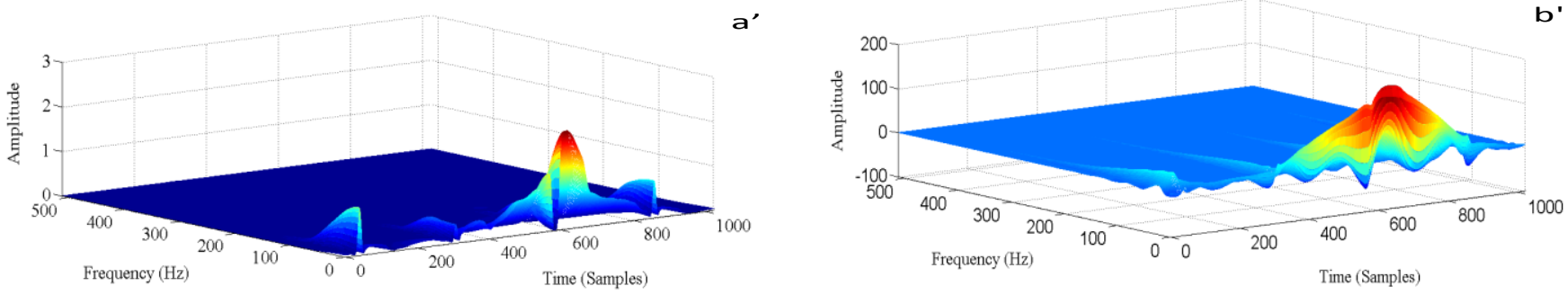

Figure.7. Periodogram (a and a') and Choi-williams (b and b') time-frequency images of an abnormal ECG signal with cu ventricular tachyarrhythmia

The figure 8 presents the time-frequency images of the abnormal ECG signal obtained from a patient with malignant ventricular arrhythmia (figure 3). The QRS complexes in this signal are wide and abnormal, while the $\mathrm{T}$ wave has disappeared. The signal seems to have an irregularity and is changing over time. The Periodogram parametric timefrequency images show a good localization of QRS complex and absence of $\mathrm{T}$ waves. All frequency components in the signal of Fig. 3 are clearly revealed by the Periodogram technique (fig $8 \mathrm{a}$ and $8 \mathrm{a}^{\prime}$ ). However, The figures $8 \mathrm{~b}$ and $8 \mathrm{~b}^{\prime}$ obtained from the application of the Choi-williams nonparametric technique present the interference terms which hide some useful information. The Choi-williams technique can't track the changes of the frequency components the QRS complex of the abnormal signal in the case the malignant ventricular arrhythmia. The high performance of the PE technique is clearly demonstrated in the figure $8\left(a\right.$ and $\left.a^{\prime}\right)$. We note from the results of the figure 8 that the parametric Periodogram technique are better than the non parametric Choi-williams technique for analyzing this delicate ECG abnormality.

The Periodogram parametric technique allow us to identify many types of abnormal ECG signals and reveal changes of frequency from the detection of the $\mathrm{T}$ waves and the QRS complexes. This parametric technique seems to be a good approach for analyzing normal and abnormal ECG signals compared to the non-parametric time-frequency techniques. 


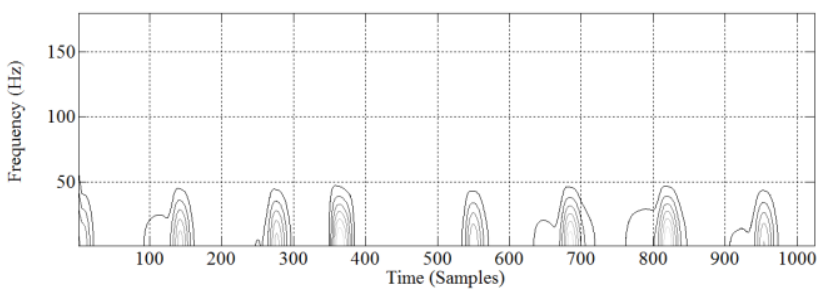

$a^{\prime}$

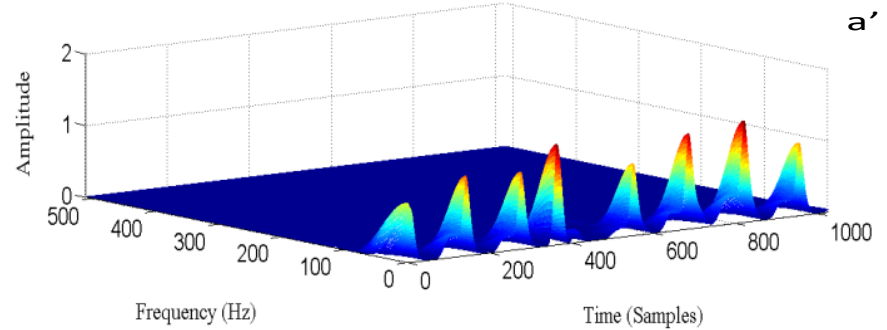

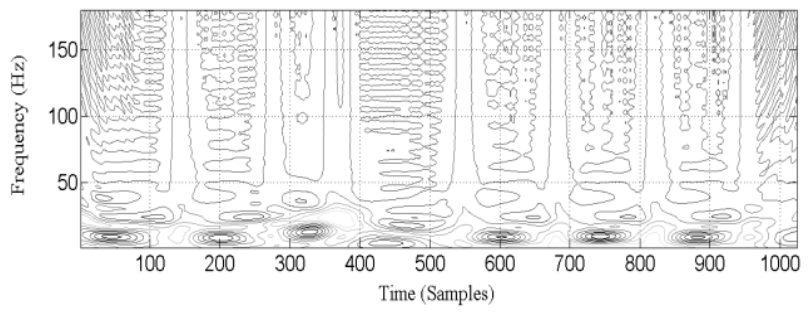

b

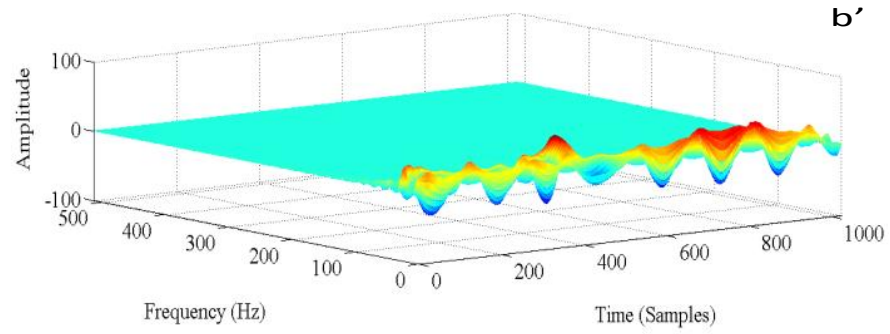

Figure.8 : Periodogram (a and a') and Choi-williams , (b and b') time-frequency images of an abnormal ECG signal with malignant ventricular arrhythmia

\section{CONCLUSION}

This paper presents a comparative study of some parametric and non parametric techniques. These time-frequency techniques are applied to normal and abnormal biomedical ECG signals. The abnormalities treated in this work, are cu ventricular tachyarrhythmia and malignant ventricular arrhythmia.

For the cu ventricular tachyarrhythmia case, the parametric technique Periodogram allows us to detect clearly the transients and QRS complexes with good resolution in the timefrequency plan. For malignant ventricular arrhythmia case, the obtained results expose the ability and the effectiveness of the parametric technique Periodogram to reveal the non-stationary behavior of this type of abnormal signals. The parametric technique can track changes in the frequency components of each QRS complex in time. The results obtained in this work, show the performance of the parametric technique Periodogram for analyzing biological signals such as ECG signal compared to others parametric and non-parametric time-frequency techniques.

\section{REFERENCES}

[1] P. de Chazal, Maraia O' Dwyer, Richard B. Reilly, "Automatic classification of heartbeats using ECG morphology and heartbeat interval features", IEEE Transactions on Biomedical Engineering 51 (7) pp. 1196-1206, 2004.

[2] G Bortolan, Christian Brohet, Sergio Fusaro, "Possibilities of using neural networks for ECG classification", Journal of Electrocardiology 29 Suppl:10-16, 1996.

[3] M.T. Özgen, "Extension of the Capon's spectral estimator to timefrequency analysis and to the analysis of polynomial-phase signals". Signal Process. 83 (3), pp. 575-592, 2003

[4] F. Castanié, Spectral Analysis Parametric and Non-Parametric Digital Methods (Ltd, 2006).

[5] S. Elouaham, R. Latif, A. Dliou, E. Aassif, B. Nassiri " Analyse et comparaison d'un signal ECG normal et bruité par la technique tempsfrequence parametrique de capon," Conference Mediterranéene sur l'Ingenierie Sure des Systemes Complexes [MISC'11, ENSA Agadir, Maroc, Mai 2010].

[6] S. Elouaham, R. Latif, A. Dliou, F. M. R. Maoulainine, M. Laaboubi, “ analysis of biomedical signals by the empirical mode decomposition and parametric time-frequency techniques" [International Symposium on security and safety of Complex Systems, may 2012, Agadir, Morocco].

[7] P. Goncalves F. Auger, P. Flandrin. Time-frequency toolbox, 1995.

[8] P. Flandrin, N. Martin, M. Basseville, Methodes temps- frequence. Suppl. Trait. Signal 9, 1992, pp. 77-147.

[9] Labib M. Khadra, "The smoothed pseudo-Wigner ville distribution in speech processing ", Int. J. Electronics, Vol. 65, No 4, pp. 743-755, 1988.

[10] R. Latif, E. Aassif, G. Maze, A. Moudden, B.Faiz, "Determination of the group and phase velocities from time-frequency representation of Wigner-Ville", Journal of Non Destructive Testing \& Evaluation International, Vol. 32, 7, pp. 415-422, 1999.

[11] R. Latif, E. Aassif, G. Maze, D.Decultot, A. Moudden, B. Faiz, “ Analysis of the circumferential acoustic waves backscattered by a tube using the time-frequency representation of wigner-ville ", Journal of Measurement Science and Technology, Vol. 11, 1, pp. 83-88, 2000.

[12] R. Latif, E. Aassif, A. Moudden, B. Faiz, "High resolution timefrequency analysis of an acoustic signal backscattered by a cylindrical shell using a Modified Wigner-Ville representation", Meas. Sci. Technol.14, pp. 1063-1067, 2003.

[13] R. Latif, E. Aassif, A. Moudden, B. Faiz, G. Maze , 'The experimental signal of a mullayer structure analysis by the time-frequency and spectral methods," NDT\&E International, Vol. 39, Issue 5, pp. 349-355, 2006.

[14] R.Latif , M. Laaboubi , E. Aassif , G. Maze. "Détermination de l'épaisseur d'un tube élastique à partir de l'analyse temps-fréquence de Wigner-Ville", Journal Acta-Acustica, Vol 95, Number 5, pp. 843-848, 2009.

[15] A. Dliou, R.Latif, M. Laaboubi, F. M. R. Maoulainine, "Arrhythmia ECG Signal Analysis using Non Parametric Time-Frequency Techniques" International Journal of Computer Applications (0975 8887) Vol 41- No.4, 2012.

[16] A. Dliou, R.Latif, M. Laaboubi, M. Laaboubi, F. M. R. Maoulainine, "Abnormal ECG Signal Analysis using Non Parametric TimeFrequency Techniques" Arabien Journal of Science engineering, July 2012 (Accepted).

[17] H. Choi, and W. Williams. "Improved time-frequency representation of multicomponent signals using exponential kernels", IEEE Trans. on Acoustics, Speech and Signal Processing, vol. 37, pp. 862-871. 1989.

[18] Physiobank, Physionet, Physiologic signal archives for biomedical research, http://www.physionet.org/physiobank. 